\title{
On the Relation between Energy Efficiency and Spectral Efficiency of Multiple-Antenna Systems
}

\author{
Jing Jiang, Member, IEEE, Mehrdad Dianati, Member, IEEE, \\ Muhammad Ali Imran, Senior Member, IEEE, Rahim Tafazolli, Senior Member, IEEE, \\ and Yan Chen, Member, IEEE.
}

\begin{abstract}
Motivated by increased interests in energy efficient communication systems, the relation between energy efficiency (EE) and spectral efficiency (SE) for multiple-input multipleoutput (MIMO) systems is investigated in this paper. To provide insight into the design of practical MIMO systems, we adopt a realistic power model, as well as consider both independent Rayleigh fading and semicorrelated fading channels. We derive a novel and closed-form upper bound for the system EE as a function of SE. This upper bound exhibits a great accuracy for a wide range of $\mathrm{SE}$ values, and thus can be utilized for explicitly assessing the influence of SE on EE, and analytically addressing the EE optimization problems. Using this tight EE upper bound, our analysis unfolds two EE optimization issues: Given the number of transmit and receive antennas, an optimum value of $\mathrm{SE}$ is derived such that the overall $\mathrm{EE}$ can be maximized; Given a specific value of SE, the optimal number of antennas is derived for maximizing the system EE.
\end{abstract}

Index Terms-Energy efficiency, Spectral efficiency, Multipleantenna systems, Rayleigh fading channels, Energy efficiency optimization.

\section{INTRODUCTION}

Multiple-input multiple-output (MIMO) systems using multiple antennas have drawn considerable attention, as they can greatly improve spectral efficiency (SE) and link reliability in wireless communications [1]. However, the use of multiple antennas will also lead to increased energy consumption for the extra circuit and signal processing. To this end, the traditional SE metric is not adequate for providing insight on designing practical MIMO systems, if the system energy efficiency (EE) is of interest. Furthermore, due to the increasing global concern about the energy consumption, innovative technologies are required to improve the overall $\mathrm{EE}$ in wireless systems [2].

Maximizing EE and SE are generally two conflicting objectives; but they can be linked and balanced through their relationship as shown in [3]. For fading channels, calculations of the ergodic capacity require to take expectations concerning a random channel matrix. The problem of defining an explicit expression for $\mathrm{EE}$ as a function of SE requires an expression

Manuscript received November 20, 2012; revised February 10, 2013; accepted February 21, 2013.

J. Jiang, M. Dianati, M.A. Imran and R. Tafazolli are with the Centre for Communication Systems Research, Department of Electronic Engineering, University of Surrey, GU2 7XH, Surrey, U.K. (Email: \{Jing.Jiang, M.Dianat, M.Imran, R.Tafazolli\}@ surrey.ac.uk)

Y. Chen is with Huawei Technologies Co. Ltd., Shanghai, China. (Email: eeyanchen@huawei.com)

This work has been done within a joint project, supported by Huawei Tech. Co. Ltd, China. for the inverse function of the capacity, and therefore is a mathematically challenging task. The first attempt to analyze the relation of $\mathrm{EE}$ and $\mathrm{SE}$ is given in [4], where an EE approximation is derived according to the first and the second derivatives of the capacity equation. Using the same approximation method, the work is then extended to relay networks in [5] and [6], where a full duplex mode at the relay is considered in [5] and half duplex considered in [6]; the work is also extended to virtual-MIMO networks in [7] and cognitive radio networks in [8]. These approximations of EE are accurate in the low SE region but largely inaccurate otherwise. In addition, the circuit energy consumption, which is often not negligible in practical wireless systems, is not considered in the aforementioned papers. Most recently, a more accurate approximation on the relation between EE and SE for MIMO channels is presented in [9] and refined in [10]: The approximation is in a closed-form expression for independent and identically distributed (i.i.d.) Rayleigh fading channels, but the expression cannot be extended to correlated fading channels and the potential EE optimization issues are not addressed. Different from the existing work, our contributions in this paper are three-fold:

- We derive a simple and closed-form upper bound (UB) for EE as a function of SE, for both i.i.d Rayleigh fading and semicorrelated fading channels. The EE UB exhibits a very good accuracy for a wide range of SE values.

- We analyze the relation between EE and SE, and the effect of increasing the number of antennas on the overall EE. To assist practical system design, a realistic power model including both transmit and circuit power consumption is considered.

- An optimum value of SE is derived for which the overall $\mathrm{EE}$ is maximized when the number of transmit and receive antennas are fixed; In addition, for a given value of SE, the optimal number of antennas is derived such that the overall $\mathrm{EE}$ is maximized.

The rest of this paper is organized as follows. Section II specifies the system model. An accurate UB for the capacity is derived in Section III and that for EE is derived in Section IV. EE optimization issues are investigated in Section V. Simulation results are in Section VI, and Section VII gives a conclusion.

\section{SySTEM MOdeL}

We consider a point-to-point MIMO communication system with $t$ transmit and $r$ receive antennas. We define $n=$ 
$\max \{t, r\}$ and $m=\min \{t, r\}$. The complex transmitted signal vector is denoted by $\boldsymbol{x} \in \mathbb{C}^{t}$, and its total power is represented by $P$ regardless of the number of antennas, i.e. $\mathbb{E}\left[\boldsymbol{x}^{\dagger} \boldsymbol{x}\right]=P$, where $\boldsymbol{x}^{\dagger}$ denotes the transpose conjugate of $\boldsymbol{x}$. The received signal $\boldsymbol{y} \in \mathbb{C}^{r}$ is then given by $\boldsymbol{y}=\boldsymbol{H} \boldsymbol{x}+\boldsymbol{n}$, where the channel matrix $\boldsymbol{H} \in \mathbb{C}^{r \times t}$ is complex Gaussian distributed [11]. The noise vector $\boldsymbol{n}$ is a zero-mean complex $r$-dimensional additive white Gaussian noise (AWGN) with variance $N$ per dimension, i.e. $\boldsymbol{n} \sim \mathcal{C N}_{r}\left(0, N \boldsymbol{I}_{r}\right)$. The average signal-to-noise ratio (SNR) is $\rho=P / N$. We assume that perfect channel state information (CSI) is known at the receiver but unknown to the transmitter side.

In a practical setting, the total power of a transmitter such as a base station (BS) is not equal to $P$, but includes various elements such as the power for radio frequency (RF) circuitry, baseband unit, or power amplifier. In general, the total power has a nearly-linear relation with both the transmit power $P$ and the antenna number $t$ [2]. Consequently, a linear power model has been abstracted for the $2 \times 2$ antenna setting and various types of BSs in [2]. We adopt this power model, i.e. the total power is

$$
P_{\text {tot }}=t\left(\xi P / t+P_{c}\right)=\xi P+t P_{c},
$$

where $P_{c}$ is the load-independent circuit power at the minimum nearly-zero output power, and $\xi$ is the scaling factor of the load-dependent power. Note that the scaling over signal load (i.e. the value of $\xi$ ) largely depends on the type of the transmitter.

\section{ERGODIC CAPACITY OF MIMO: EXPLICIT EXPRESSION VS. UB}

The ergodic capacity of the MIMO system specified in Section II is obtained by averaging the channel capacity with respect to the random channel matrix $\boldsymbol{H}$ [12], i.e.

$$
\begin{aligned}
C_{\mathrm{MIMO}}= & \mathbb{E}\left[\log _{2} \operatorname{det}\left(\boldsymbol{I}_{m}+\frac{\rho}{t} \boldsymbol{\Xi}\right)\right] \quad \text { bits } / \mathrm{s} / \mathrm{Hz}, \\
& \text { with } \boldsymbol{\Xi}= \begin{cases}\boldsymbol{H H}^{\dagger}, & t \geq r \\
\boldsymbol{H}^{\dagger} \boldsymbol{H}, & t<r,\end{cases}
\end{aligned}
$$

where the random matrix $\boldsymbol{\Xi} \in \mathbb{C}^{m \times m}$ and $\boldsymbol{I}_{m}$ denotes an $m \times m$ identity matrix.

\section{A. Independent and Identically Distributed MIMO Channels}

We first consider an i.i.d. Rayleigh-fading case. Without loss of generality, the channel matrix is assumed to have unit variance entries, i.e. $\boldsymbol{H} \sim \mathcal{C N}_{r, t}\left(0, \boldsymbol{I}_{r} \otimes \boldsymbol{I}_{t}\right)$, where $\otimes$ denotes the Kronecker product of matrices. Then $\boldsymbol{\Xi}$ has a complex central Wishart distribution with $n$ degrees of freedom and covariance matrix $\boldsymbol{I}_{m}$, i.e. $\boldsymbol{\Xi} \sim \mathcal{C W}_{m}\left(n, \boldsymbol{I}_{m}\right)$. In the following lemma, we give a preliminary result of UB on $C_{\text {MIMO. }}$

Lemma 1: If $\boldsymbol{H} \sim \mathcal{C} \mathcal{N}_{r, t}\left(0, \boldsymbol{I}_{r} \otimes \boldsymbol{I}_{t}\right)$, i.e. for an i.i.d. Rayleigh fading MIMO channel, the UB of the ergodic capacity in bits/s/Hz, denoted as $\widehat{C}_{\text {MIMO }}$, is given by

$$
\widehat{C}_{\mathrm{MIMO}}=m \log _{2}\left(\frac{\rho}{t}\right)+\log _{2}(m !)+\log _{2}\left[\mathcal{L}_{m}^{n-m}(-t / \rho)\right],
$$

where $m$ ! denotes the factorial of $m$, and $\mathcal{L}_{m}^{\alpha}(x)$ is the Laguerre polynomial of order $m$ [13] (eq. 8.970.1)

$$
\mathcal{L}_{m}^{\alpha}(x)=\frac{1}{m !} e^{x} x^{-\alpha} \frac{d^{m}}{d x^{m}}\left(e^{-x} x^{m+\alpha}\right) .
$$

In particular, for the case when $\mathrm{SNR}$ is high (i.e. $P \gg N$ ), we have

$$
\widehat{C}_{\text {MIMO }}=m \log _{2}(\rho / t)+\log _{2}[n ! /(n-m) !] .
$$

Proof: The closed-form UB for $C_{\text {MIMO }}$ in (3) was first derived in [14] (Theorem 2). To obtain (5), we implement $\mathcal{L}_{m}^{n-m}(0)=$ $n ! /[m !(n-m) !]$ [13] (eq. 8.973.3).

\section{B. Spatially Semicorrelated MIMO Channels}

In practice, the MIMO channels are often correlated due to limited angular spreads or restrictions on array sizes at either end. The effects of spatial semicorrelation can be reflected by using the Kronecker model

$$
\boldsymbol{H}=\left\{\begin{array}{cl}
\boldsymbol{\Phi}^{1 / 2} \boldsymbol{G}, & \text { if } t \geq r \\
\boldsymbol{G} \boldsymbol{\Phi}^{1 / 2}, & \text { if } t<r,
\end{array}\right.
$$

where $\boldsymbol{G} \sim \mathcal{C N}_{r, t}\left(0, \boldsymbol{I}_{r} \otimes \boldsymbol{I}_{t}\right)$ and $\boldsymbol{\Phi}$ denotes the spatial correlation matrix at either the transmitter or receiver end. Note that here we focus our research on the semicorrelated fading channels where the correlation occurs at the end with the minimum number of antennas, in order to reflect the practical mobile communication environments where insufficient array spacing exists at mobile terminals [15]. In this case, $\boldsymbol{\Xi}$ has a complex central Wishart distribution $\Xi \sim \mathcal{C} \mathcal{W}_{m}(n, \boldsymbol{\Phi})$.

Lemma 2: For a spatially semicorrelated Rayleigh fading MIMO channel, i.e. $\boldsymbol{H}$ is given by (6), under the condition of high SNR, the ergodic capacity in bits/s/Hz is upper bounded as

$$
\widehat{C}_{\mathrm{MIMO}}=m \log _{2}\left(\frac{\rho}{t}\right)+\log _{2}\left[\frac{n !}{(n-m) !}\right]+\log _{2} \operatorname{det}(\boldsymbol{\Phi}) \text {. }
$$

Proof: See Appendix A.

Comparing (7) with (5), the capacity reduction because of the spatial semicorrelation is $-\log _{2} \operatorname{det}(\boldsymbol{\Phi})$ bits/s/Hz at high SNR. Note that, the results (5) and (7) provide simple and tight UBs of the ergodic capacity, which are less accurate than the explicit expression (2), but have the advantage of being express in closed-form and can be used to evaluate the relation between $\mathrm{EE}$ and SE for MIMO systems.

\section{ENERGY EFFICIENCY ANALYSIS OF MIMO CHANNELS}

We use the well known metric of the system achievable $\mathrm{EE}$ as the number of information bits transmitted per Joule of energy, i.e. $E_{\mathrm{MIMO}}=S_{\mathrm{MIMO}} / P_{\text {tot }}$ in bits/Joule, where $S_{\mathrm{MIMO}}$ denotes SE of the system. For a system with unit bandwidth, we have $S_{\text {MIMO }}=C_{\text {MIMO }}$ in (2). The choice of $S_{\text {MIMO }}$ and

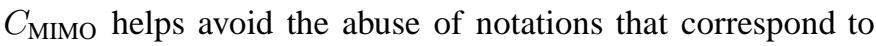
the functions of $E_{\mathrm{MIMO}}$ and $P$ [6]. According to (1) and (2), both $P_{\text {tot }}$ and $S_{\text {MIMO }}$ are related to $P$. Thus the impact of $S_{\text {MIMO }}$ on $E_{\text {MIMO }}$ can be expressed as

$E_{\mathrm{MIMO}}=S_{\mathrm{MIMO}}\left[\xi P+t P_{c}\right]^{-1}=S_{\mathrm{MIMO}}\left[\xi f^{-1}\left(C_{\mathrm{MIMO}}\right)+t P_{c}\right]^{-1}$, 
where $f^{-1}: C_{\text {MIMO }} \in[0,+\infty) \mapsto P \in[0,+\infty)$ is the inverse function of $C_{\text {MIMO }}$. Equation (8) indicates that obtaining an explicit expression of $E_{\mathrm{MIMO}}$ boils down to finding an explicit formula for $f^{-1}\left(C_{\mathrm{MIMO}}\right)$. However, due to the random Rayleigh channel realizations in $(2), f^{-1}\left(C_{\text {MIMO }}\right)$ cannot be straightforwardly formulated. One feasible approach would be to use the closed-form UB of $C_{\text {MIMO }}$ in Lemma 1 or Lemma 2 to find a solution to $f^{-1}\left(C_{\mathrm{MIMO}}\right)$. This approach will help us obtain an EE UB as a function of SE, which can be utilized for tracking the EE of MIMO systems, and thus will be discussed in this section.

\section{A. EE UB for I.I.D. MIMO Channels}

We use the closed-form UB of $C_{\text {MIMO }}$, and according to the definition of EE, we obtain a closed-form UB for the system $\mathrm{EE}$ as follows.

Proposition 1: If $\boldsymbol{H} \sim \mathcal{C N}_{r, t}\left(0, \boldsymbol{I}_{r} \otimes \boldsymbol{I}_{t}\right)$, i.e. for an i.i.d. Rayleigh-fading MIMO channel, the UB for the number of bits transmitted per Joule of energy, denoted by $\widehat{E}_{\mathrm{MIMO}}$, is given by

$$
\widehat{E}_{\mathrm{MIMO}}=\frac{S_{\mathrm{MIMO}}}{t P_{c}+t \xi N \exp \left\{\frac{S_{\mathrm{MIMO}} \ln 2}{m}+\frac{1}{m} \ln \left[\frac{(n-m) !}{n !}\right]\right\}} .
$$

Proof: According to (8), when $S_{\text {MIMO }}$ is small (correspondingly $P$ is small), the value of $E_{\mathrm{MIMO}}$ is dominated by the circuit power $P_{c}$. In contrast, $E_{\mathrm{MIMO}}$ is more sensitive and needs a more accurate expression when $P$ is large. We thus consider the high SNR case and employ (5) to find an approximated solution to $f^{-1}\left(C_{\mathrm{MIMO}}\right)$ as follows

$$
f^{-1}\left(C_{\mathrm{MIMO}}\right)=t N 2^{S_{\mathrm{MIMO}} / m+\log _{2}\left[\frac{(n-m) !}{n !}\right] / m} .
$$

Substituting (10) into (8), we obtain (9). This completes the proof.

\section{B. EE UB for Spatially Semicorrelated MIMO Channels}

We now consider semicorrelated Rayleigh fading channels, and use the capacity UB in Lemma 2 for finding an explicit solution of $P=f^{-1}\left(C_{\mathrm{MIMO}}\right)$. Solving $P$ from (7), and inserting the solution in (8), we thus obtain an EE UB for spatially semicorrelated MIMO channels as shown in the following.

Proposition 2: For a spatially semicorrelated Rayleigh fading MIMO channel, i.e. $\boldsymbol{H}$ is given by (6), the number of bits transmitted per Joule of energy can be upper bounded by

$$
\widehat{E}_{\mathrm{MIMO}}=\frac{S_{\mathrm{MIMO}}}{t P_{c}+t \xi N \exp \left\{\frac{S_{\mathrm{MIMO}} \ln 2}{m}+\frac{1}{m} \ln \left[\frac{(n-m) !}{n ! \operatorname{det} \boldsymbol{\Phi}}\right]\right\}} .
$$

Example (Exponential Correlation Model): We take a common model that often used to effectively quantify the spatial correlation, i.e. the exponential correlation model [16], as an example. More specifically, the correlation matrix can be constructed using a single coefficient $\phi$ with $|\phi| \leq 1$,

$$
\boldsymbol{\Phi}_{i j}= \begin{cases}\phi^{|j-i|}, & i \leq j \\ \phi^{|j-i|}, & i>j\end{cases}
$$

where $\bar{x}$ means the complex conjugate of $x$. This exponential model may approximate the correlation in a uniform linear array under rich scattering conditions [16]. We consider $\boldsymbol{\Phi}$ as a $l \times l$ correlation matrix, and from (12), we have

$$
\operatorname{det} \boldsymbol{\Phi}=(1-\phi \bar{\phi})^{l-1}=\left(1-|\phi|^{2}\right)^{l-1},
$$

where $l$ could equal either $t$ or $r$ depending on the correlation at which side. Inserting (13) in (11), we will obtain the EE UB of the Rayleigh fading channels with exponential semicorrelation.

Therefore, for both i.i.d. and spatially semicorrelated MIMO channels, the EE UB $\widehat{E}_{\text {MIMO }}$ has been obtained in a simple closed-form expression. The EE UB is very valuable to realistic MIMO systems: It can help the system explicitly track the influence of SE on EE, and also address the EE optimization problems as will be discussed in the following section.

\section{ENERGY EFFICIENCY OPTIMIZATION ISSUES}

In this subsection, we optimize the overall EE performance of MIMO systems, by taking the factors, various values of SE as well as different numbers of transmit and receive antennas, into consideration. The optimization criterion is based on the tight EE UB derived above.

\section{A. Optimum SE to Maximize EE}

As stated above, for a given number of $t$ and $r$, there exists a certain region of $\mathrm{SE}$ corresponding to a better EE. In the following proposition, the optimum value of $\mathrm{SE}$ is derived to maximize the overall EE.

Proposition 3: For a spatially semicorrelated Rayleigh fading MIMO channel with correlation matrix $\Phi$ and $t$ transmit and $r$ receive antennas, the optimum value of $\mathrm{SE}$ for which the system EE is maximized, denoted by $S_{\mathrm{MIMO}}^{*}$, is given by

$$
S_{\text {MIMO }}^{*}=\frac{m}{\ln (2)}+\frac{m}{\ln (2)} \omega\left\{\frac{P_{c}}{\xi N \mathrm{e}} \exp \left(-\frac{1}{\mathrm{~m}} \ln \left[\frac{(\mathrm{n}-\mathrm{m}) !}{\mathrm{n} ! \operatorname{det} \boldsymbol{\Phi}}\right]\right)\right\},
$$

where $\omega\{\cdot\}$ is the Lambert $\omega$-function satisfying $\omega(z) e^{\omega(z)}=$ $z$. Let det $\boldsymbol{\Phi}=1, S_{\text {MIMO }}^{*}$ is obtained for i.i.d. Rayleigh fading MIMO channels.

Proof: We first consider spatially semicorrelated Rayleigh fading channels, as the results of optimum SE for i.i.d. fading channels can be obtained as a special case. One can apply the method for convex optimization: From (11), the first-order derivative of $\widehat{E}_{\mathrm{MIMO}}$ with respect to $S_{\mathrm{MIMO}}$ is given by

$$
\begin{gathered}
\frac{d \widehat{E}_{\mathrm{MIMO}}\left(S_{\mathrm{MIMO}}\right)}{d S_{\mathrm{MIMO}}}=\left[t P_{c}+t \xi N \exp \left\{\frac{S_{\mathrm{MIMO}} \ln 2}{m}+\frac{1}{m} \ln \left[\frac{(n-m) !}{n ! \operatorname{det} \boldsymbol{\Phi}}\right]\right\}\right]^{-1} \\
-\frac{t \xi N S_{\mathrm{MIMO}} \ln (2) \exp \left\{\frac{S_{\mathrm{MIMO}} \ln (2)}{m}+\frac{1}{m} \ln \left[\frac{(n-m) !}{n ! \operatorname{det} \boldsymbol{\Phi}}\right]\right\}}{m\left[t P_{c}+t \xi N \exp \left\{\frac{S_{\mathrm{MIMO}} \ln 2}{m}+\frac{1}{m} \ln \left[\frac{(n-m) !}{n ! \operatorname{det} \boldsymbol{\Phi}}\right]\right\}\right]^{2}} .
\end{gathered}
$$

By setting $\frac{d \widehat{E}_{\text {MIMO }}\left(S_{\text {MIMO }}\right)}{d S_{\text {MIMO }}}=0$, one can obtain a unique closedfrom solution $S_{\text {MIMO }}^{*} \in[0,+\infty)$. We introduce Lambert $\omega$ function [17], and $S_{\mathrm{MIMO}}^{*}$ is expressed as shown in (14). As a special case of (14), i.e. $\operatorname{det} \boldsymbol{\Phi}=1$, we obtain $S_{\text {MIMO for the }}^{*}$ case of i.i.d. fading channels. This completes the proof. 


\section{B. Optimization of the Numbers of Antennas}

As shown in (9) and (11), for a specific value of SE, different numbers of transmit and receive antennas result in different levels of EE. Finding out the optimal number of antennas (both $t$ and $r$ ) such that the overall EE is maximized, is practically appealing and thus analyzed here. As it is not always possible to place many antennas at the receiver in practice, $t \geq r$ (i.e. $m=r$ ) is considered in this subsection.

Proposition 4: If $\boldsymbol{H} \sim \mathcal{C N}_{r, t}\left(0, \boldsymbol{I}_{r} \otimes \boldsymbol{I}_{t}\right)$ with $t \geq r$, given a target $S_{\text {MIMO }}$, for maximizing the overall EE, the optimal numbers of transmit and receive antennas, denoted by $t^{*}$ and $r^{*}$, are given by

$$
\begin{aligned}
& t^{*}=r^{*}= \begin{cases}\lfloor\tau\rfloor, & \text { if } \widehat{E}_{\mathrm{MIMO}}(\lfloor\tau\rfloor) \geq \widehat{E}_{\mathrm{MIMO}}(\lceil\tau\rceil) \\
\lceil\tau\rceil, & \text { if } \widehat{E}_{\mathrm{MIMO}}(\lfloor\tau\rfloor)<\widehat{E}_{\mathrm{MIMO}}(\lceil\tau\rceil),\end{cases} \\
& \text { where } \tau=\frac{S_{\mathrm{MIMO}} \ln (2)}{2}\left\{\omega\left[\left(\frac{4 \xi N \mathrm{Ne}}{P_{c} S_{\mathrm{MIMO}} \ln (2)}\right)^{-\frac{1}{2}}\right]\right\}^{-1},
\end{aligned}
$$

where $\lfloor\cdot\rfloor$ and $\lceil\cdot\rceil$ denote the floor and ceiling integer functions, respectively. Proof: To find the maximum EE, treat $t$ and $r$ as continuous variables. There are two natural restrictions of $t \geq 1$ and $r \geq 1$. Considering $m=r$, from (9), we obtain

$$
\widehat{E}_{\mathrm{MIMO}}=\frac{S_{\text {MIMO }}}{t P_{c}+t \xi N \exp \left\{\frac{S_{\mathrm{MIMO}} \ln 2}{r}+\frac{1}{r} \ln \left[\frac{(t-r) !}{t !}\right]\right\}} \text {. }
$$

We can observe that for a specific $t$, increasing $r$ will result in a higher value of $E_{\mathrm{MIMO}}$. It is because the system capacity increases with $r$, as shown in (5), and no extra transmit power is required. Thus, as the EE metric in (17) is a linear increasing function with respect to $r$ and $t \geq r \geq 1$, the maximum value of EE is located at $r^{*}=t$. Let $r=t$, we have $\widehat{E}_{\mathrm{MIMO}}(t)$. In addition, we implement Stirling's formula, i.e. $\ln n !=n \ln n-$ $n$, for approximation and extending factorials to non-integer values of argument. Then the first-order derivative of $\widehat{E}_{\mathrm{MIMO}}(t)$ concerning $t$ is

$$
\frac{d \widehat{E}_{\mathrm{MIMO}}(t)}{d t}=\frac{S_{\text {MIMO }}\left\{\xi N \text { e } 2^{\frac{S_{\text {MIMO }}}{t}} S_{\text {MIMO }} \ln (2)-t^{2} P_{c}\right\}}{t^{2}\left\{\xi N \text { e } 2^{\frac{S_{\text {MIMO }}}{t}}+t P_{c}\right\}^{2}} .
$$

Setting (18) to zero for $t$ will yield the non-integer optimal solution $\tau$. Note that under the restriction of $t \geq 1$, the solution of $\tau$ is unique. As $t^{*}$ and $r^{*}$ need to be integers, we substitute $t=\lfloor\tau\rfloor$ or $t=\lceil\tau\rceil$ to $\widehat{E}_{\mathrm{MIMO}}(t)$, compare $\widehat{E}_{\mathrm{MIMO}}(\lfloor\tau\rfloor)$ and $\widehat{E}_{\mathrm{MIMO}}(\lceil\tau\rceil)$, and thus obtain $t^{*}$ and $r^{*}$ as shown in (16).

We then analyze the case of spatially semicorrelated MIMO channels, which is more complicated than the i.i.d. case, as $\operatorname{det} \boldsymbol{\Phi}$ is not independent to the number of antennas. We thus choose the exponential correlation model with coefficient $\phi$ and obtain the following proposition.

Proposition 5: For an exponentially semicorrelated Rayleigh fading MIMO channel with correlation coefficient $\phi$ and $t \geq r$, given a target $S_{\mathrm{MIMO}}$, to maximize the overall $\mathrm{EE}$, the optimal

$$
\begin{aligned}
& t^{*} \text { and } r^{*} \text { are } \\
& t^{*}=r^{*}=\left\{\begin{array}{l}
\lfloor\tau\rfloor, \text { if } \widehat{E}_{\mathrm{MIMO}}(\lfloor\tau\rfloor) \geq \widehat{E}_{\mathrm{MIMO}}(\lceil\tau\rceil) \\
\lceil\tau\rceil, \text { if } \widehat{E}_{\mathrm{MIMO}}(\lfloor\tau\rfloor)<\widehat{E}_{\mathrm{MIMO}}(\lceil\tau\rceil),
\end{array}\right. \\
& \text { where } \tau=\frac{S_{\mathrm{MIMO}}}{2 / \ln (2)}\left\{\omega\left[\left(\frac{4 \xi N \mathrm{e}\left[S \ln (2)+\ln \left(1-|\phi|^{2}\right)\right]}{S_{\mathrm{MIMO}}^{2} \ln (2)^{2} P_{c}\left(1-|\phi|^{2}\right)}\right)^{-\frac{1}{2}}\right]\right\}^{-1} .
\end{aligned}
$$

Proof: Considering the exponential correlation with coefficient $\phi$ and considering $m=r$, from (11) and (13), we have

$$
\begin{aligned}
& \widehat{E}_{\mathrm{MIMO}}(t)= \\
& \frac{S_{\mathrm{MIMO}}}{t P_{c}+t \xi N \exp \left\{\frac{S_{\mathrm{MIMO}} \ln 2}{t}-\frac{1}{t} \ln (t !)-\frac{t-1}{t} \ln \left(1-|\phi|^{2}\right)\right\}} .
\end{aligned}
$$

Treat $t$ as a continuous variable. The first-order derivative of $\widehat{E}_{\mathrm{MIMO}}(t)$ concerning $t$ is given by

$$
\begin{aligned}
& \frac{d \widehat{E}_{\text {MIMO }}(t)}{d t} \\
& =\frac{S_{\text {MIMO }}\left\{\xi N \mathrm{e} 2 \frac{S_{\text {MIMO }}}{t}\left(1-|\phi|^{2}\right)^{-\frac{t-1}{t}}\left[S_{\text {MIMO }} \ln (2)+\ln \left(1-|\phi|^{2}\right)\right]-t^{2} P_{c}\right\}}{t^{2}\left\{\xi N \mathrm{e} 2^{\frac{S_{\text {MIMO }}}{t}}\left(1-|\phi|^{2}\right)^{-\frac{t-1}{t}}+t P_{c}\right\}^{2}} \\
& \approx \frac{S_{\text {MIMO }}\left\{\xi N \mathrm{e} 2^{\frac{S_{\text {MIMO }}}{t}}\left[S_{\text {MIMO }} \ln (2)+\ln \left(1-|\phi|^{2}\right)\right]-t^{2}\left(1-|\phi|^{2}\right) P_{c}\right\}}{t^{2}\left(1-|\phi|^{2}\right)\left\{\xi N \mathrm{e} 2^{\frac{S_{\text {MIMO }}}{t}}\left(1-|\phi|^{2}\right)^{-1}+t P_{c}\right\}^{2}} .
\end{aligned}
$$

Setting (21) to zero, we get the non-integer solution $\tau$; substituting $t=\lfloor\tau\rfloor$ or $t=\lceil\tau\rceil$ to $\widehat{E}_{\mathrm{MIMO}}(t)$, we thus obtain the optimal $t^{*}$ and $r^{*}$ as shown in (19). When the correlation coefficient $\phi=0$ (i.e., no correlation happened), (19) reduces to (16), and therefore (19) still holds for the i.i.d. fading channels.

For a practical MIMO communication system with a specific type of base station, given any number of transmit and receive antennas, Proposition 3 can help the system explicitly find out the optimum value of SE, such that the system is able to save energy to the maximum extent. In addition, for a system having a specific SE requirement, Proposition 4 and Proposition 5 show the optimal numbers of $t$ and $r$ which can be used for the system to maximize the overall EE. These findings are therefore particularly valuable and appealing to realistic wireless communication systems.

\section{Vi. Simulation Results and Discussions}

In this section, we present the EE performance and the relation between EE and SE for MIMO channels by using both simulation and analytical results. The simulation results are obtained from the Monte Carlo method for random channel realizations. Without loss of generality, we assume the noise variance $N$ is normalized to unity. The values of the power model parameters for the transmitter are assumed as $\xi=2.4$ and $P_{c}=130$, which are adopted from [2] where a Macro type of transmitter is considered.

Firstly, we consider i.i.d. Rayleigh-fading MIMO channels, and verify the accuracy of the EE UB given by Proposition 1 in Fig. 1 (a). This figure shows that the EE UBs are quite tight to the simulation results for the entire range of SE, regardless of the number of antennas. Using the EE UBs, Fig. 1 (b) shows 
how the load-independent circuit power (i.e. $t P_{c}$ ) and loaddependent power (i.e. $\xi P$ ) components impact the overall EE. When only load-dependent power is considered, a monotonic tradeoff relationship between EE and SE exists, i.e., increasing $\mathrm{SE}$ leads to a decrease in EE. With the total supply power, as illustrated in both Fig. 1 (a) and (b), there is a certain region of SE that corresponds to better EE: When SE is low, the overall EE is dominated by the constant circuit power. As SE increases, the load-dependent power contributes more to $P_{\text {tot }}$; thus, EE increases up to a certain level but finally decreases.

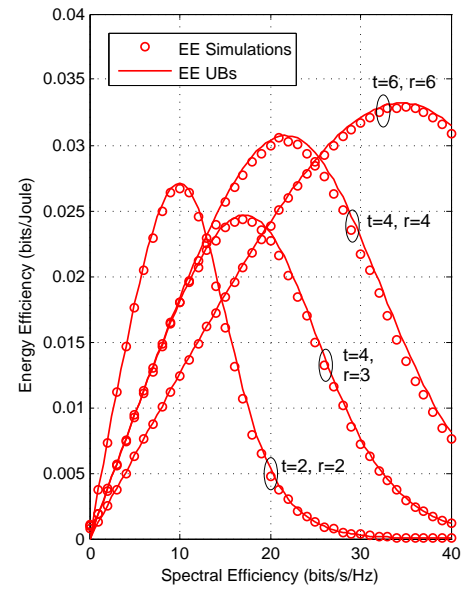

(a)

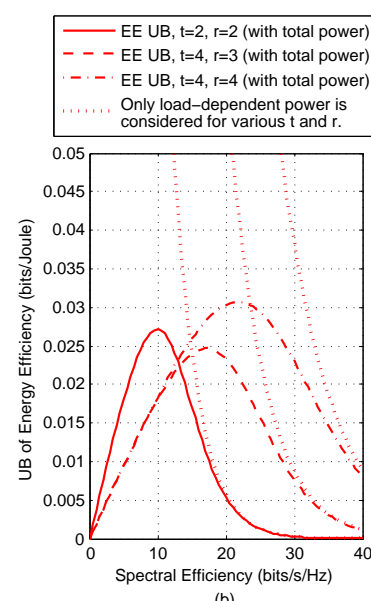

Fig. 1. The relation between EE and SE for i.i.d. MIMO channels with $t$ transmit and $r$ receive antennas (EE simulation results and UBs are shown in (a), and the effects of load-dependent power on EE are in (b).)

Fig. 2 shows the EE performance for Rayleigh-fading MIMO channels with exponential correlation at the receiver side considering the same numbers of antennas as in Fig. 1 (a). The correlation coefficient $\phi$ is set to 0.5 , and the UBs are plotted using Proposition 2. Compared to the i.i.d. case in Fig. 1 (a), both EE simulations and UBs in Fig. 2 are slightly lower due to the effects from channel correlation. Nevertheless, for both cases, the EE UBs are very tight to the simulation results for the whole range of SE, which is important for the optimization purposes. Taking practical small numbers of antennas as examples, by using Proposition 3, we have the optimum value of SE equal to $9.65 \mathrm{bits} / \mathrm{s} / \mathrm{Hz}$ for $t=r=2$, and $16.54 \mathrm{bits} / \mathrm{s} / \mathrm{Hz}$ for $t=4, r=3$; their accuracies are demonstrated by simulation results in Fig. 2 .

Fig. 3 shows EE of exponentially semicorrelated Rayleighfading MIMO channels as a function of correlation coefficient $\phi$ at $S_{\text {MIMO }}=20 \mathrm{bits} / \mathrm{s} / \mathrm{Hz}$. As expected, EE decreases significantly with an increase in correlation coefficient $\phi$, particularly for larger $t$ and $r$ as the correlation is worse. But the case of $t=r=6$ makes an exception: It is because that when SE equals $20 \mathrm{bits} / \mathrm{s} / \mathrm{Hz}$, circuit power (i.e. $t P_{c}$ ) dominates EE (as shown in Fig. 2), and EE under this case is more tolerant to the channel correlation. In contrast, when the transmit power dominates, EE will be more sensitive to the value of $\phi$.

Using the accurate EE UBs, Fig. 4 (a) demonstrates the effects of varying the numbers of antennas on the relation between EE and SE for Rayleigh-fading MIMO channels.

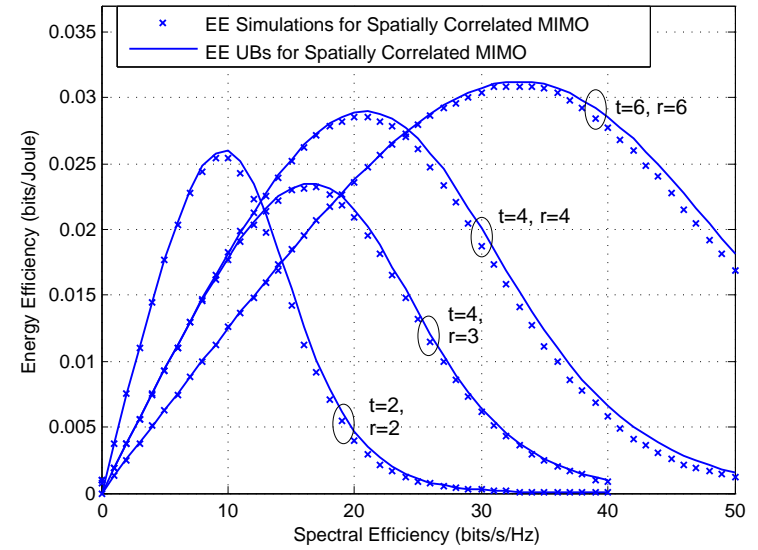

Fig. 2. EE simulations and UBs for spatially correlated Rayleigh-fading channels with $t$ transmit and $r$ receive antennas (The receiver-side correlations are exponential correlations with $\phi=0.5$. )

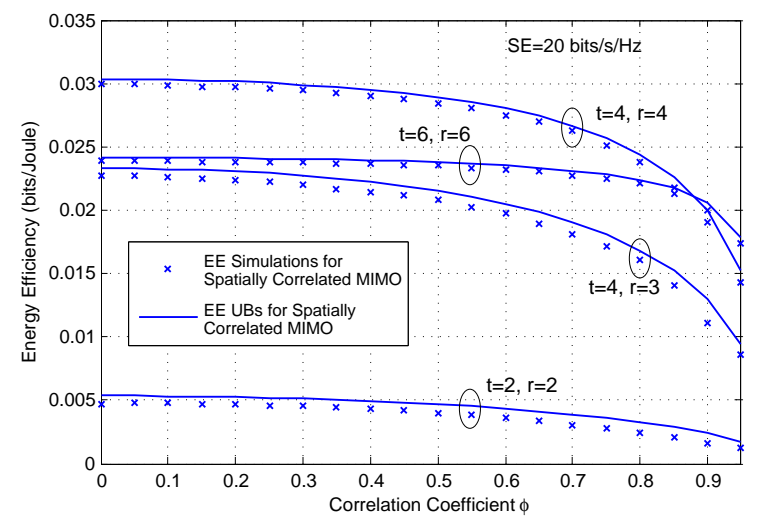

Fig. 3. The EE performance as a function of coefficient $\phi$ for exponentially semicorrelated Rayleigh-fading MIMO channels at $S_{\mathrm{MIMO}}=20 \mathrm{bits} / \mathrm{s} / \mathrm{Hz}$.

$\phi$ is set to 0.5 for the case of correlated fading channels. When the number of antennas are fixed, there exists an optimum value of SE corresponding to a maximized EE, as illustrated in Proposition 3. In addition, for certain values of SE, Fig. 4 (b) shows the optimal numbers of antennas $\left(t^{*}=r^{*}\right)$ for maximizing the overall EE, using Proposition 4 and Proposition 5 for i.i.d. fading and semicorrelated fading channels, respectively. Every step changing in Fig. 4 (b) are almost in correspondence with Fig. 4 (a) for a maximized EE, which also demonstrates the accuracy of Proposition 4 and Proposition 5.

\section{CONCLUSION}

This paper has investigated the EE performance and the influence of SE on EE in MIMO systems over Rayleigh fading channels, while considering a realistic power model. We have derived a simple and closed-form UB for the system ergodic capacity, and based on which we have proposed a novel and closed-form UB for EE as a function of SE. The effects of various fading channels have been considered, including i.i.d. Rayleigh fading channels and semicorrelated fading channels. The EE UB exhibits a very good accuracy for a wide range of SE values, and thus is utilized for analytically addressing 

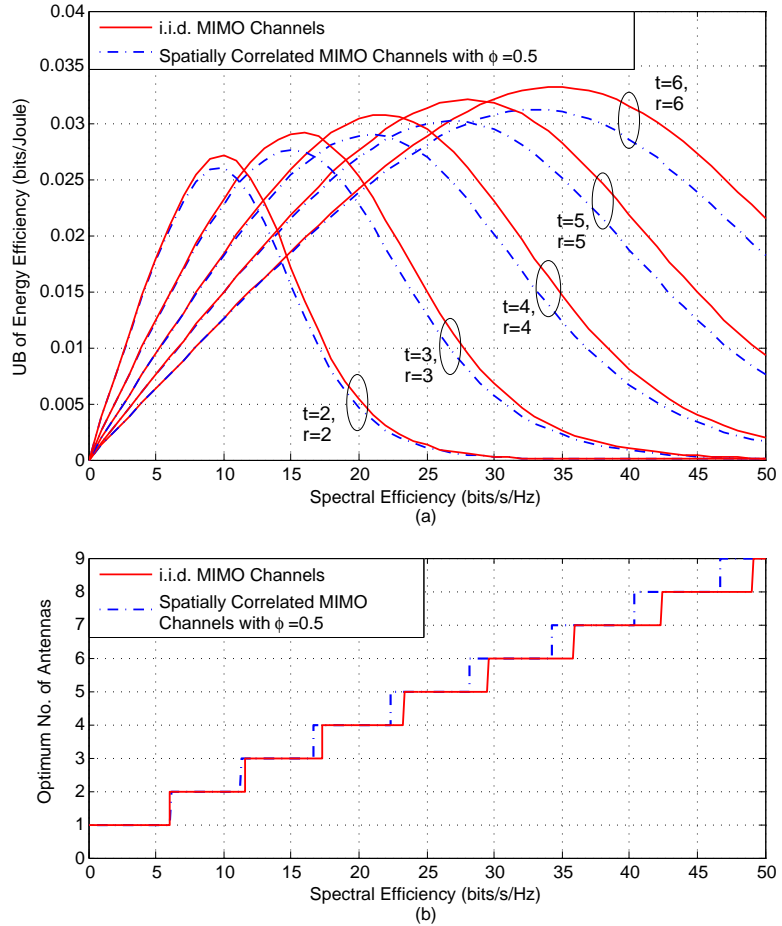

Fig. 4. The effects of varying the numbers of antennas on the relation between EE and SE for Rayleigh-fading MIMO channels (The correlation coefficient $\phi$ is set to 0.5 for the case of exponentially semicorrelated channels.)

the EE optimization issues. Our results have demonstrated that, compared to the existing work only considering transmit power (where a monotonic trade-off relation exists between $\mathrm{EE}$ and $\mathrm{SE}$ ), there is a certain region of $\mathrm{SE}$ that corresponds to better EE performance. The optimum value of SE has been derived such that the overall EE is maximized when the number of transmit and receive antennas is given. In addition, our results have shown that different numbers of antennas result in different levels of EE. Given a target SE, the optimal number of antennas has been derived for maximizing the EE performance.

\section{ACKNOWLEDGMENT}

This work has been done within a joint project, supported by Huawei Tech. Co. Ltd, China.

\section{APPENDIX A}

\section{PROOF OF LEMMA 2}

If $\boldsymbol{Y} \sim \mathcal{C W}_{m}(n, \boldsymbol{\Phi})$, the density function of $\boldsymbol{Y}$ is [18]

$$
p_{\boldsymbol{Y}}(\boldsymbol{Y})=\frac{1}{\Gamma_{m}(n)}[\operatorname{det}(\boldsymbol{\Phi})]^{-n}[\operatorname{det}(\boldsymbol{Y})]^{n-m} \operatorname{etr}\left(-\boldsymbol{\Phi}^{-1} \boldsymbol{Y}\right), \boldsymbol{Y}>0
$$

where $\operatorname{tr}(\boldsymbol{A})$ denotes the trace of $\boldsymbol{A}$ and $\operatorname{etr}(\boldsymbol{A})=\exp \operatorname{tr}(\boldsymbol{A})$. $\Gamma_{m}(n)=\pi^{m(m-1) / 2} \prod_{j=0}^{m-1} \Gamma(n-j)$ is the multivariate gamma function and $\Gamma(\cdot)$ is the gamma function. From the density of $\boldsymbol{Y}$, we have

$$
\begin{aligned}
& \mathbb{E}[\operatorname{det}(\boldsymbol{Y})]= \\
& \frac{1}{\Gamma_{m}(n)}[\operatorname{det}(\boldsymbol{\Phi})]^{-n} \cdot \int_{\boldsymbol{Y}>0}[\operatorname{det}(\boldsymbol{Y})]^{n-m+1} \operatorname{etr}\left(-\boldsymbol{\Phi}^{-1} \boldsymbol{Y}\right) d \boldsymbol{Y} .
\end{aligned}
$$

Make the change of variate $\boldsymbol{Y}=\boldsymbol{\Phi}^{1 / 2} \boldsymbol{Z} \boldsymbol{\Phi}^{1 / 2}$, with Jacobian $(d \boldsymbol{Y})=[\operatorname{det}(\boldsymbol{\Phi})]^{m}(d \boldsymbol{Z})$. Then we have

$$
\begin{aligned}
& \mathbb{E}[\operatorname{det}(\boldsymbol{Y})] \\
& =\frac{1}{\Gamma_{m}(n)}[\operatorname{det}(\boldsymbol{\Phi})]^{-n} \int_{\boldsymbol{Z}>0}[\operatorname{det}(\boldsymbol{Z})]^{n-m+1}[\operatorname{det}(\boldsymbol{\Phi})]^{n+1} \operatorname{etr}(-\boldsymbol{Z}) d \boldsymbol{Z} \\
& =\frac{1}{\Gamma_{m}(n)}[\operatorname{det}(\boldsymbol{\Phi})]^{-n}[\operatorname{det}(\boldsymbol{\Phi})]^{n+1} \Gamma_{m}(n+1) \\
& =\operatorname{det}(\boldsymbol{\Phi}) \prod_{j=0}^{m-1}(n-j) .
\end{aligned}
$$

Applying Jensen's inequality to (2) and at high SNR, we have $C_{\mathrm{MIMO}} \leq \log _{2} \mathbb{E}\left[\operatorname{det}\left(\boldsymbol{I}+\frac{\rho}{t} \boldsymbol{\Xi}\right)\right] \approx \log _{2} \mathbb{E}\left[\operatorname{det}\left(\frac{\rho}{t} \boldsymbol{\Xi}\right)\right]$.

As $\boldsymbol{\Xi} \sim \mathcal{C W}_{m}(n, \boldsymbol{\Phi})$, we let $\boldsymbol{\Xi}=\boldsymbol{Y}$. Using (24), we get (7).

\section{REFERENCES}

[1] J. Jiang, J. S. Thompson, and H. Sun, "A singular-value-based adaptive modulation and cooperation scheme for virtual-MIMO systems," IEEE Trans. Vehicular Technology, vol. 60, no. 6, pp. 2495-2504, July 2011.

[2] G. Auer, V. Giannini, C. Desset, I. Godor, P. Skillermark, M. Olsson, M. Imran, D. Sabella, M. Gonzalez, O. Blume, and A. Fehske, "How much energy is needed to run a wireless network?" IEEE Wireless Communications, vol. 18, no. 5, pp. 40-49, Oct. 2011.

[3] G. Li, Z. Xu, C. Xiong, C. Yang, S. Zhang, Y. Chen, and S. Xu, "Energyefficient wireless communications: tutorial, survey, and open issues," IEEE Wireless Communications, vol. 18, no. 6, pp. 28-35, Dec. 2011.

[4] S. Verdu, "Spectral efficiency in the wideband regime," IEEE Transactions on Information Theory, vol. 48, no. 6, pp. 1319-1343, Jun. 2002.

[5] X. Cai, Y. Yao, and G. B. Giannakis, "Achievable rates in low-power relay links over fading channels," IEEE Trans. Commun., vol. 53, no. 1, pp. 184-194, 2005.

[6] J. Gomez-Vilardebo, A. Perez-neira, and M. Najar, "Energy efficient communications over the AWGN relay channel," IEEE Trans. Wireless Commun., vol. 9, no. 1, pp. 32-37, Jan. 2010.

[7] J. Jiang, M. Dianati, M. Imran, and Y. Chen, "Energy efficiency and optimal power allocation in virtual-MIMO systems," in IEEE Vehicular Technology Conference, Quebec City, Canada, Sep. 2012, pp. 1-6.

[8] F. Haider, C.-X. Wang, X. Hong, H. Harald, D. Yuan, and E. Hepsaydir, "Spectral-energy efficiency tradeoff in cognitive radio networks with peak interference power constraints," in IEEE 13th ICCT, Sept. 2011, pp. $368-372$.

[9] F. Heliot, O. Onireti, and M. Imran, "An accurate closed-form approximation of the energy efficiency-spectral efficiency trade-off over the MIMO Rayleigh fading channel," in 2011 IEEE ICC Workshops, June 2011, pp. 1-6.

[10] F. Heliot, M. Imran, and R. Tafazolli, "On the energy efficiency-spectral efficiency trade-off over the MIMO Rayleigh fading channel," IEEE Trans. Communications, vol. 60, no. 5, pp. 1345-1356, May 2012.

[11] H. Sun, D. I. Laurenson, and C.-X. Wang, "Computationally tractable model of energy detection performance over slow fading channels," IEEE Communications Letters, vol. 14, no. 10, pp. 924-926, Oct. 2010.

[12] J. Jiang, J. Thompson, H. Sun, and P. Grant, "Performance assessment of virtual multiple-input multiple-output systems with compress-andforward cooperation," IET Communications, vol. 6, no. 11, pp. 1456 $-1465,242012$

[13] I. S. Gradshteyn and I. M. Ryzhik, Table of Integrals, Series, and Products, Seventh Edition. San Diego, CA: Academic Press, 2007.

[14] A. Grant, "Rayleigh fading multi-antenna channels," EURASIP Journal on Applied Signal Processing (Special Issue on Space-time Coding Part I), vol. 2002, no. 3, pp. 316-329, Mar. 2002.

[15] P. J. Smith, S. Roy, and M. Shafi, "Capacity of MIMO systems with semicorrelated flat fading," IEEE Trans. Information Theory, vol. 49, no. 10 , Oct. 2003.

[16] S. Chatzinotas, M. Imran, and R. Hoshyar, "On the multicell processing capacity of the cellular MIMO uplink channel in correlated rayleigh fading environment," IEEE Trans. Wireless Commun., vol. 8, no. 7, pp. 3704-3715, July 2009.

[17] C.-L. Chen, W. Stark, and S.-G. Chen, "Energy-bandwidth efficiency tradeoff in MIMO multi-hop wireless networks," IEEE Journal on Selected Areas in Communications, vol. 29, no. 8, pp. 1537-1546, Sep. 2011.

[18] A. K. Gupta and D. K. Nagar, Matrix Variate Distributions. Boca Raton, Fla, USA: Chapman \& Hall/CRC, 2000. 